\title{
Classical critical dynamics in quadratically driven Kerr resonators
}

\author{
Wouter Verstraelen (10 and Michiel Wouters \\ Theory of Quantum and Complex Systems, University of Antwerp, B-2610 Wilrijk, Belgium
}

(Received 14 February 2020; accepted 19 March 2020; published 21 April 2020)

\begin{abstract}
Driven-dissipative Kerr lattices with two-photon driving are experimentally relevant systems known to exhibit a symmetry-breaking phase transition, which belongs to the universality class of the thermal Ising model for the parameter regime studied here. In this work, we perform finite-size scaling of this system as it is quenched to the transition, and the dynamical critical exponent is found to be compatible with $z \approx 2.18$, corresponding with metropolis dynamics in classical simulations. Furthermore, we show that the Liouvillian gap scales with the same exponent, similar to scaling of the Hamiltonian gap at quantum phase transitions in closed systems.
\end{abstract}

DOI: 10.1103/PhysRevA.101.043826

\section{INTRODUCTION}

Thermal phase transitions in classical systems connected to a heath bath, as well as quantum phase transitions in closed quantum systems, are well understood $[1,2]$. Very recently, a strong interest has emerged in the critical behavior of open quantum systems [3], for example, in a driven-dissipative context, fueled by the realization that such systems may gain importance as a workhorse in quantum technologies [4].

The central object in such systems is not the free energy, as in thermal phase transitions, or the Hamiltonian, as in quantum phase transitions, but the Liouvillian superoperator [5,6]. This object can exhibit a rich phenomenology [7], as both the quantum phase transition and the thermal phase transitions are, in principle, limiting cases corresponding to a vanishing dissipation rate or coupling with a thermal bath, respectively. It appears, nonetheless, that thermal behavior may emerge in this setting more often, as long as sufficiently strong dissipation is present. Some recent results on this emergence of classical critical behavior were obtained regarding the phase of free exciton-polariton condensates [8-11] and the Dicke model [12-14], and include also non-Markovian extensions [15]. Nevertheless, a number of genuinely nonequilibrium phase transitions are also known to be possible in this context [16-20].

One of the promising platforms in the study of dissipative phase transitions are arrays of quadratically (two-photon) driven Kerr cavities, objects originally suggested in the quest for noise-resilient quantum codes [21-25] and for which building blocks have currently been experimentally realized with superconducting circuits [26,27]. In this system, it has been explicitly demonstrated numerically that its symmetrybreaking phase transition [28] exhibits a crossover from quantum to classical critical behavior regarding the steady-state properties for increasing dissipation in the form of singlephoton losses. In particular, the system was found to belong to the universality class of the quantum or thermal Ising model, respectively [29,30]. The crossover between these two spin models has also been subject to more general recent studies on the dynamics [31] and thermalization mechanism [32].
Here, we are concerned with extending the conclusions in the large-dissipation limit to the dynamical aspects of the phase transition. In particular, we are interested in the dynamical critical exponent $z$ that relates the correlation time, and in closed quantum systems the Hamiltonian gap, to the control parameter and the correlation length. Near a critical point, the correlation time diverges, leading to the occurrence of critical slowing down. Some current works have addressed the latter effect in a dissipative context for optical bistability (a first-order dissipative phase transition) [33-35], fermionic lattices [36], optical lattice clocks [37], and miscellaneous spin lattices $[38,39]$.

For our study of the quadratically driven Kerr lattice, we will use two separate approaches. First, we address the occurrence of the Kibble-Zurek (KZ) effect. Because of the critical slowing down, domains are formed when the control parameter (photon driving in our case) is tuned through the transition at finite speed. According to a dynamical scaling hypothesis based on the $\mathrm{KZ}$ effect [40,41], we are able to extract $z$ from square lattices subject to a linear quench and obtain $1.9<z<2.3$. Second, we study the slow relaxation dynamics to the steady state. This latter timescale is known to correspond directly to the inverse Liouvillian gap [6]. We here find scaling with $1.8<z<2.3$. The fact that both values are consistent implies that the Liouvillian gap exhibits the same scaling relations as the Hamiltonian gap in closed quantum systems.

The two-dimensional classical Ising model is known as a paradigmatic example of the second-order phase transition, both in terms of its historical importance as for its pedagogical value $[1,42,43]$. In numerical simulations, it is typically implemented with a somewhat heuristic update rule, which is not uniquely defined by the steady-state properties. Moreover, different update rules applied to the same model result in different values of $z$. The most well known of these rules is the single-site update rule or Metropolis algorithm, which is consistent with experimental observations of the critical dynamics in an iron film [44]. The same value of $z$ is found in the Hohenberg-Halperin model A dynamics [45], as well as in $\phi^{4}$ theory [46]. 
We find here that the value of $z$ extracted from quadratically driven Kerr resonators is indeed consistent with the value $z_{m} \approx 2.18$ from the Metropolis algorithm and model $\mathrm{A}$ dynamics. This correspondence between our nonequilibrium critical dynamics and model A dynamics is in line with Ref. [11] on critical dynamics of a driven-dissipative BoseHubbard model.

\section{QUADRATICALLY DRIVEN PHOTONIC LATTICES}

The Hamiltonian of a $d$-dimensional lattice of nonlinear bosonic resonators with nearest-neighbor hopping and coherent two-photon driving, rotating at half the driving frequency, is

$$
\begin{aligned}
\hat{H}= & \sum_{i=1}-\Delta \hat{a}_{i}^{\dagger} \hat{a}_{i}+\frac{U}{2} \hat{a}_{i}^{\dagger 2} \hat{a}_{i}^{2}+\frac{G}{2} \hat{a}_{i}^{\dagger 2}+\frac{G^{*}}{2} \hat{a}_{i}^{2} \\
& -\sum_{\langle i j\rangle} \frac{J}{2 d}\left(\hat{a}_{i}^{\dagger} \hat{a}_{j}+\hat{a}_{j}^{\dagger} \hat{a}_{i}\right),
\end{aligned}
$$

where $\hat{a}_{i}\left(\hat{a}_{i}^{\dagger}\right)$ annihilates (creates) a photon at site $i$, and the last summation runs over nearest-neighbor pairs [28]. $G$ is the two-photon driving amplitude, $U$ the Kerr nonlinearity, $J$ the hopping strength, and $\Delta$ the detuning between half the driving frequency and the cavity frequency. Furthermore, dissipation in the form of single-photon losses can, under Born-Markov approximation, be described with a jump operator $\sqrt{\gamma} \hat{a}$, leading to a Lindblad master equation,

$$
\frac{\partial \hat{\rho}}{\partial t}=\mathcal{L} \hat{\rho},=-i[\hat{H} \hat{\rho}]+\gamma \sum_{j} \hat{a}_{j} \hat{\rho} \hat{a}_{j}^{\dagger}-\frac{\gamma}{2}\left\{\hat{a}_{j}^{\dagger} \hat{a}_{j}, \hat{\rho}\right\} .
$$

Generally, two-photon losses can also be present in this system, but as suggested by analytic [29] and numeric [30] arguments, these have little qualitative influence on the critical behavior [47] while being computationally constraining, so that we do not include this process explicitly in this work.

A first study on system (2) was performed on the meanfield level in [28] and predicted the occurrence of spontaneous breaking of the $\mathbb{Z}_{2}$ symmetry when increasing $G$. Recently, this prediction was confirmed in a similar parameter regime with the additional finding that the transition belongs to the universality class of the thermal Ising model [30]. In the weakloss limit, by contrast, the transition belongs to the quantumIsing universality class, and the system can be explicitly mapped on an $X Y$-spin model [29]. For negative $J$, antiferromagnetic behavior emerges [48]. A brief look at the single-site problem [21] provides a simple picture to understand these behaviors: in each individual site, there are two metastable coherent-state solutions with displacement $\pm \alpha_{0}$ (with a purely imaginary value in our considered case in absence of twophoton losses) that can be easily interpreted as $|\uparrow\rangle$ and $|\downarrow\rangle$ spin states. Alignment of these coherent states in neighboring sites then corresponds to ferromagnetism, whereas a more random distribution $+\alpha_{0}$ and $-\alpha_{0}$ corresponds to classical paramagnetic behavior, and maximal disalignment means antiferromagnetism. In the quantum paramagnet, the sites are highly entangled into superpositions of the coherent states.

The former studies were all restricted to steady-state properties of the transition. Here we extend the results of Ref. [30] obtained with the Gaussian trajectory approach (GTA) to the dynamical properties of the phase transition.

According to the quantum trajectory framework [3,5], an open quantum system can be described as a stochastic average over quantum trajectories $\left\{|\psi\rangle_{s}\right\}$, corresponding to singleshot experimental realizations. Crucially, even though the full evolution (2) does not break the symmetry, individual trajectories $\left\{|\psi\rangle_{s}\right\}$ can. The GTA [49,50] adds a Gaussian ansatz to this formalism. This means that every trajectory $|\psi\rangle_{s}$ of an $N$-mode system is characterized only by the coherent displacements $\left\{\alpha_{s, i}\right\}$ and the anomalous $\left\{u_{s, i j}\right\}$ and normal $\left\{v_{s, i j}\right\}$ quantum correlations where $1 \leqslant i, j \leqslant N$. Explicitly, these coefficients are defined as

$$
\begin{aligned}
\alpha_{s, i} & =\left\langle\psi_{s}\left|\hat{a}_{i}\right| \psi_{s}\right\rangle \\
u_{s, i j} & =\left\langle\psi_{s}\left|\hat{a}_{i} \hat{a}_{j}\right| \psi_{s}\right\rangle-\alpha_{s, i} \alpha_{s, j} \\
v_{s, i j} & =\left\langle\psi_{s}\left|\hat{a}_{i}^{\dagger} \hat{a}_{j}\right| \psi_{s}\right\rangle-\alpha_{s, i}^{*} \alpha_{s, j} .
\end{aligned}
$$

The Gaussian ansatz thus reduces the complexity of each trajectory to quadratic as a function of system size. In Ref. [30], the corresponding GTA equations were derived explicitly for the quadratically driven photonic lattice. By solving these to the steady state, emergence of an ordered phase was first witnessed by a macroscopic occupation of the $k_{0}$ mode. Furthermore, the quantity $\bar{\alpha}=\Im \frac{1}{N} \sum_{i} \alpha_{i}$ becomes a suitable real-valued order parameter akin to a magnetization of which the distribution (sampled by values $\bar{\alpha}_{s}$ ) changes from monomodal in the paramagnetic (disordered) phase to bimodal in the ferromagnetic (ordered) phase. Using finite-size scaling of the Binder cumulant, which quantifies this behavior [51], the critical exponent $v=1$ was extracted, indicating that the transition belongs to the universality class of the classical Ising model, as well as the critical value $G_{c} \approx 0.86$ for the parameters $U=\gamma=J=1, \Delta=-1$.

For the dynamical numerical studies in this work, we are able to evolve these GTA equations with the same time step as the static case [30] $\left(h=10^{-4}\right.$ for the Euler-Maruyama method which coincides with Milstein's method [52]) because our interest is focused on timescales that are slower than those corresponding to individual Hamiltonian or dissipation terms.

\section{KIBBLE-ZUREK SCALING AT A LINEAR QUENCH}

When a parameter in a thermodynamic system is slowly varied, the adiabatic theorem assures that the system remains at all times in an equilibrium state at constant entropy. The minimal ramp time for equilibrium to be preserved is given by the relaxation time. When a parameter is quenched through a second-order phase transition, the relaxation time diverges, adiabaticity always breaks down, and domains with different values of the symmetry-breaking order parameter are formed. This mechanism is known as the Kibble-Zurek mechanism [40]. Originally introduced in a cosmological context [53], it has been developed further mainly in condensed-matter systems, with first applications to liquid helium [54] and rotor models, where the defects are vortices [40]. More recently, the $\mathrm{KZ}$ mechanism has been applied to more generic systems where the defects take the form of domain walls $[55,56]$. 


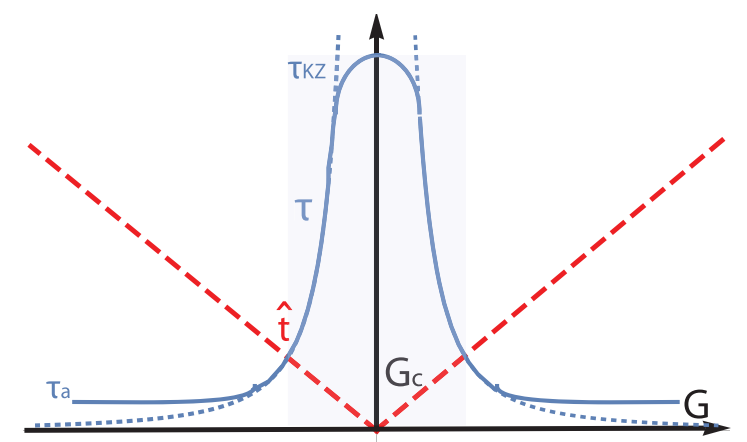

FIG. 1. The Kibble-Zurek effect: When $G$ is linearly increased, the time until $G_{c}$ is reached (red, dashed line) becomes less than the diverging correlation time $\tau$ (blue line) at $\hat{t}$. From $\hat{t}$ onwards, the dynamics freezes (blue region). In a finite system, the true value of $\tau$ (full line) only follows the universal behavior for intermediate velocities $v$ for which the crossing occurs at $\tau_{a}<\tau<\tau_{K Z}$.

Extensions to quantum phase transitions have also been developed [41,57-60].

More specifically, the $\mathrm{KZ}$ mechanism works as follows (Fig. 1). We envision a continuous quench where, starting from the steady-state solution at some value $G_{0}$ in the paramagnetic regime, $G$ is increased linearly up to $G_{c}$ in a total time $T$ :

$$
G(t)=G_{0}+v t \quad 0 \leqslant t \leqslant T,
$$

where $v=\frac{G_{c}-G_{0}}{T}$. As $G_{c}$ is approached, the correlation time diverges as $\tau \sim\left(G-G_{c}\right)^{-z \nu}$ while the correlation length scales as $\xi \sim\left(G-G_{c}\right)^{-v}$. From some point in time during the quench $T-\hat{t}$ onward, $\tau(G)>\hat{t}$ : the dynamics freezes as the time available for the dynamics drops below the correlation time. The correlation length is then unable to increase beyond $\xi_{c} \sim\left(G(T-\hat{t})-G_{c}\right)^{-v}$, setting the final domain size. Crucially, the lower $v$, the larger $G(T-\hat{t})$ and thus the larger the domains. The former arguments assume universality in an infinite lattice. An example of such domain formation after quenching with different $v$ from a given initial state is given in Fig. 2.

Focusing on the steady-state properties of a more realistic finite lattice system, there are three distinct length scales present: the lattice spacing $a=1$, correlation length $\xi$, and system size $L$. Close to criticality, one has $\xi \gtrsim a$, leading to the scaling hypothesis that all quantities can be expressed as a function of the dimensionless ratio $\xi / L$ [61]. Such an approach was also used to study system (2) in previous work [30].

For dynamic scaling, we now follow the scaling approach introduced in Ref. [41]. Three different timescales are present: $\tau_{a}$ relates to the local individual Hamiltonian processes and loss rate, $\tau$ the correlation time, and $\tau_{K Z}$ the timescale for the adiabatic evolution of the finite system. In general, universal behavior for $\xi$ and $\tau$, and hence standard Kibble-Zurek scaling, can be expected for $\tau_{a}<\hat{t}<\tau_{K Z}$ (Fig. 1). Each of these timescales is associated with velocity scales [41].

On the order of the lattice spacing [62], $v_{a} \sim J^{2} a^{-(z+1 / v)}=$ $J^{2}$ marks the transition speed between possibly microscopic dynamics and long-range universal behavior. The second scale
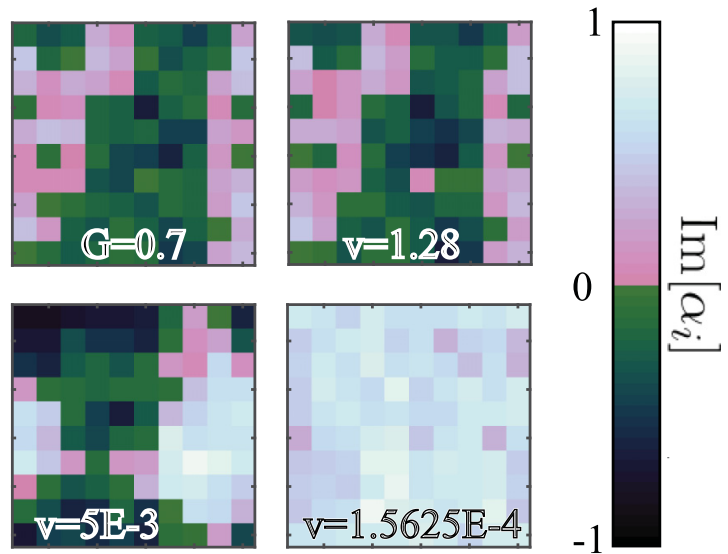

FIG. 2. During the quench protocol, $G$ is increased linearly with time from $G_{0}=0.7 J$ to $G_{c}=0.86 J$, the critical point [30]. The upper left panel shows a Monte Carlo sampling of the steady state at the $G=G_{0}$. The other panels show a sample of the final state that was evolved with different quench speeds $v$. For $v \gtrsim 1$, almost no evolution has been able to take place. For decreasing $v$, the correlations are able to spread further, until for $v \lesssim v_{k z}(L)$ the whole $10 \times 10$ lattice is correlated.

is the quench speed $v$. Third, there is the Kibble-Zurek speed $v_{K Z}(L) \sim J^{2} L^{-(z+1 / v)}$, marking the speed where domain size becomes comparable with the lattice size (below $v_{K Z}(L)$ the dynamics remain adiabatic). Now, two separate scaling functions appear for the second moment of the order parameter after the quench $(t=T)$ in different regimes [41]. If the quench is sufficiently slow for the microscopic processes to be unimportant $\left(v \lesssim v_{a}\right)$, one expects universal scaling as a function of $v / v_{K Z}$ :

$$
\left\langle\bar{\alpha}^{2}\right\rangle=L^{-2 \beta / v} f_{1}\left(v L^{z+1 / v}\right),
$$

where $\langle\cdot\rangle$ denotes a statistical expectation value over trajectories. On the other hand, if the quench is sufficiently fast for the finite size to be unimporant $\left[v \gtrsim v_{K Z}(L)\right]$, universal scaling of $v / v_{a}$ is expected, leading to

$$
\left\langle\bar{\alpha}^{2}\right\rangle=L^{-d} f_{2}\left(v^{-1}\right) .
$$

In the overlapping region $v_{k z} \lesssim v \lesssim v_{a}$, both scaling functions overlap with a power-law dependence $\left\langle\bar{\alpha}^{2}\right\rangle \propto v^{-x}$ with the exponent

$$
x=\frac{d-2 \beta / \nu}{z+1 / v} .
$$

The above arguments have extensively been verified for thermal systems, but the generic nature of arguments suggests that they should also work out of equilibrium. In order to verify the $\mathrm{KZ}$ mechanism in the two-photon driven-dissipative Hubbard model, we have simulated quenches in the amplitude of the two-photon drive of the Bose-Hubbard model from $G_{0}=0.7 \mathrm{~J}$ to $G_{c}=0.86 \mathrm{~J}$.

In Fig. 3 the extracted slow and fast scaling functions $f_{1}$ and $f_{2}$ are shown for different square lattice sizes, and collapse is observed for both when using exponents $\beta=0.125, v=$ $1, z \approx 2.18$. The former two numbers are static critical exponents corresponding to the two-dimensional (2D) thermal Ising model [30]. The value of $z$, the dynamical critical 

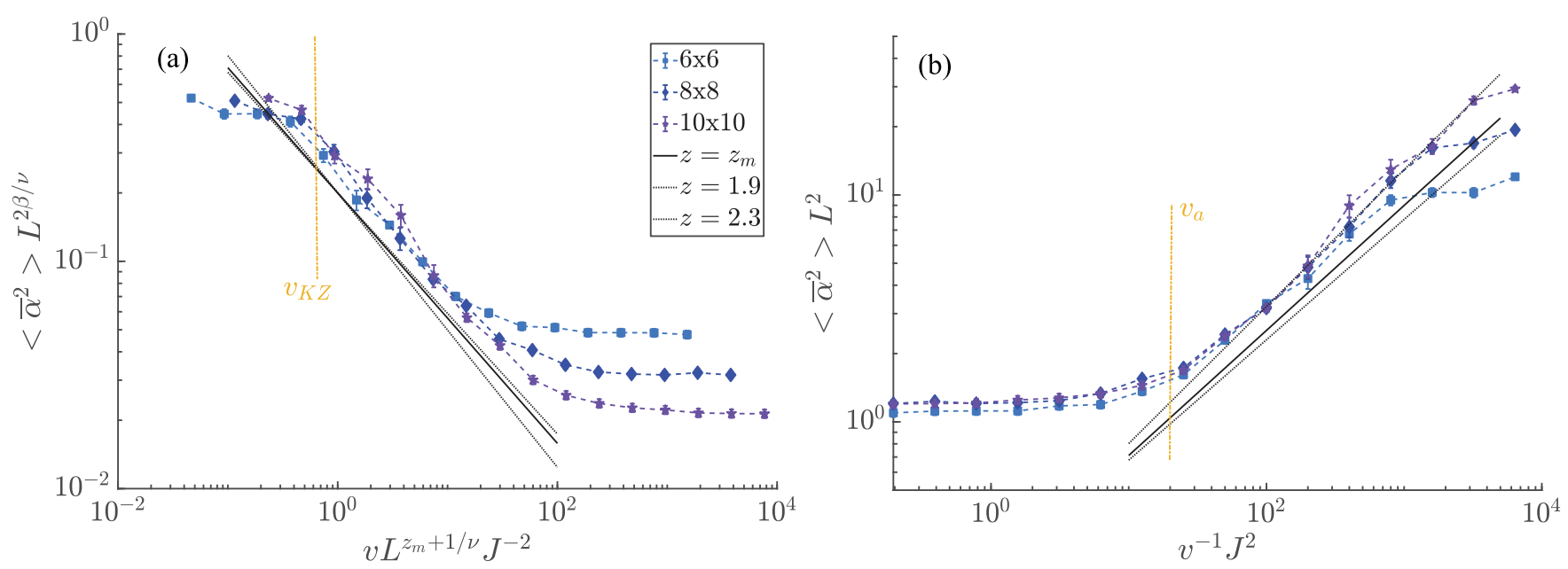

FIG. 3. Dynamic scaling functions for different system sizes showing collapse in the adiabatic $\left[f_{1},(\mathrm{a})\right]$ and diabatic limit $\left[f_{2},(\mathrm{~b})\right]$ for linear quenches from $G_{0}=0.7 \mathrm{~J}$ to $G_{c}=0.86 \mathrm{~J}$. In the intermediate regime, there is a universal power-law scaling (black line) with exponent $x=\frac{d-2 \beta / v}{z+1 / v}$, where the dimension $d=2 ; \beta=0.125, v=1$ are critical exponents of the universality class of the $2 \mathrm{D}$ classical Ising model; and $z$ is the dynamical critical exponent. Results are consistent with $z=z_{m} \approx 2.18$, the dynamical critical exponent characterizing Metropolis dynamics in the 2D Ising model; this value was also used for the finite-size scaling itself. Parameters: $U=\gamma=J=1, \Delta=-1$. For the fastest quenches $\left(v / J^{2} \geqslant 10^{-2}\right), 10^{3}$ trajectories were used and $10^{2}$ trajectories for the slower quenches. The yellow vertical lines denote approximate values for $v_{K Z}$ and $v_{a}$, marking the edges of the regime where power-law scaling is valid. We find that the prefactor in the definition of $v_{K Z}$ is of order 1 , whereas $v_{a} \approx 10 J^{2}$.

exponent, corresponds to Metropolis dynamics in this model [63]. In the intermediate regime for $v$, the predicted power law (7) is further observed, consistent with the aforementioned values of the critical exponents.

We thus have obtained strong evidence for the fact that not only the static but also the dynamical properties of the twophoton driven Bose-Hubbard model are in the Ising universality class, more precisely, they are of Metropolis dynamics.

\section{THE LIOUVILLIAN GAP SCALES WITH THE SAME EXPONENT}

In Hamiltonian systems, the dynamical exponent $z$ is further known to govern the scaling of the gap $\Delta_{H}$ [2]:

$$
\Delta_{H} \sim \xi^{-z} \& \Delta_{H} \sim\left|g-g_{c}\right|^{z v},
$$

where $g$ is the control parameter. Because system (2) studied here belongs to a classical universality class due to its drivendissipative nature, the fate of relations (8) is not a priori clear. In an open system, the dynamics are governed by the Liouvillian superoperator $\mathcal{L}$ [6]. Likewise, the timescale of slowest relaxation to the steady state is determined by the Liouvillian gap $\lambda$, defined as (minus) the real part of the first nonzero eigenvalue of $\mathcal{L}$. One can thus ask if $\Delta_{H}$ can be replaced by $\lambda$ in relations (8). If this replacement in the first relation of (8) is valid, then one must have in a finite system [61]

$$
\lambda=\xi^{-z} \tilde{f}(\xi / L)=L^{-z} f\left[L^{1 / v}\left(G-G_{c}\right)\right],
$$

where $\tilde{f}, f$ are unknown scaling functions.

In order to obtain values of $\lambda$ for different values of $G$ and $L$ numerically, we perform the following procedure in each case. After starting from a fully polarized $\left(\alpha_{j}=1 i, \forall j\right)$ state, the system is left to evolve freely. An exponential $\sim e^{-\lambda t}$ is then fitted to the slow relaxation process towards the steady state, as illustrated for a $10 \times 10$ lattice in the left panel of Fig. 4.

The validity of Eq. (9) implies a collapse of curves for different system sizes when plotting $L^{z} \lambda$ as function of $L^{1 / \nu}(G-$ $G_{c}$ ). In the right panel of Fig. 4 , we see that this is indeed the case for $v=1, z=z_{m}$, the same values as in the previous section.

Furthermore, relation (9) also directly relates $\lambda$ to control parameter $G$. In the limit of large system sizes, we indeed observe (black line on Fig. 2)

$$
\lambda \sim\left|G-G_{c}\right|^{z v}
$$

where the numerical result is especially good in the regime $\xi<L$. This means that both relations (8) are valid for the considered Liouvillian dynamics.

\section{CONCLUSIONS}

In a dissipative Bose-Hubbard model with two-photon driving, the classical Ising model can be simulated, where the role of magnetization is taken by polarization of the optical phase. Not only does the transition in this system belong to the universality class of the Ising model at equilibrium, but also the dynamical properties match as witnessed by the value of critical exponent $z$.

We have also shown that the Liouvillian gap $\lambda$ scales with the same exponent $z$, with relations very reminiscent of the scaling of Hamiltonian gaps at quantum phase transitions in closed systems. To what extent this scaling behavior is generic for open quantum systems is an interesting open problem.

It is furthermore interesting to note that there are alternatives for the metropolis in classical Ising simulations, with different values of $z$, which can converge to the steady state faster (Swedsen-Wang, Wolff) or slower (East). One may wonder 

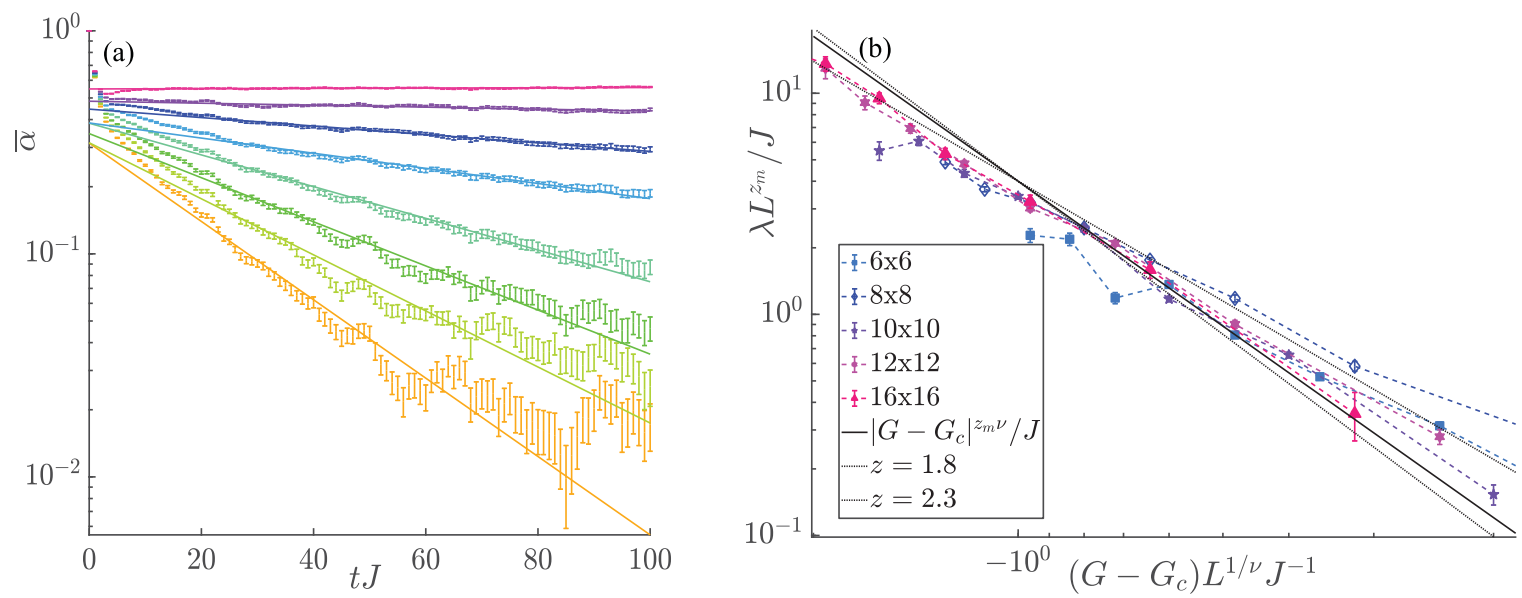

FIG. 4. (a) Error bars show the decay of $\bar{\alpha}$ to the steady-state value (0) in a $10 \times 10$ lattice after initialization in a polarized $\alpha_{j}=1 i, \forall j$ state, average over trajectories. From bottom to top, $G$ values run increment from $0.74 J$ with steps $0.02 J$ to $0.86 J$. Full lines in corresponding colors: fits $\propto e^{-\lambda t}$, with fitting from $t J=20 / 30 / 40$ onwards $(6 \times 6,10 \times 10 / 12 \times 12 / 16 \times 16)(t J=10-50$ for the $8 \times 8$ case $)$. (b) Extracted values of $\lambda$, rescaled assuming $z=z_{m}$ as a function of rescaled $G$. Especially in the $\xi<L$ regime (where the function argument less than -1 ), collapse is very clear and in agreement with power-law behavior (10). Closer to the critical point, the data collapse becomes worse. It should be noted that in this regime, the timescales become very long and the extracted decay rate may be less accurate. Moreover, the slow dynamics is more sensitive to rare events that may not be sufficiently sampled. $10^{3}$ trajectories were used in the simulations up to $12 \times 12$ lattices and $10^{2}$ for the $16 \times 16$ case.

if suitable reservoir engineering would allow simulation of these as well. This could be useful to speed up or slow down relaxation to the same steady state numerically or experimentally. A possibility would be exploiting the difference between one common bath or independent baths, which has shown to reflect at least on the decoherence time of an open quantum system [32].

It would also be interesting to see how the dynamical criticality behaves in the quantum regime, where the KibbleZurek effect can exhibit richer behavior [60], as has also been suggested for study experimentally with trapped ions [64]. Recently, also anti-Kibble-Zurek behavior was found under certain circumstances in open quantum systems $[65,66]$.

Even within a classical regime, optical simulation of the Ising model has been proposed to solve NP-hard tasks [67], including through degenerate parametric oscillators [68-70], a system with an analogous symmetry breaking to ours. To this purpose, our results also point out that, especially in absence of an all-to-all connected setup, care must be taken when driving through the transition that $v<v_{K Z}$ in order to find the true steady state (Ising ground state) and not a metastable state with Kibble-Zurek domains. Recently also implementations of the Ising model in a Kerr resonator have been proposed [71] where optical bistability in the single-photon driven case is used to map the two spin states. Unlike this situation, the $\mathbb{Z}_{2}$ symmetry is exact in Kerr resonators with the two-photon driving studied here, which might benefit the accuracy of the results of such optimization algorithms.

\section{ACKNOWLEDGMENTS}

We acknowledge stimulating discussions and comments on the manuscript from R. Rota and F. Minganti. The computational resources and services used in this work were provided by the VSC (Flemish Supercomputer Center), funded by the Research Foundation - Flanders (FWO) and the Flemish Government department EWI. Financial support from Project FWO-39532 is acknowledged.
[1] K. Huang, Statistical Mechanics (John Wiley \& Sons, New York, 2014).

[2] S. Sachdev, Quantum Phase Transitions (Cambridge University Press, Cambridge, England, 2011).

[3] H.-P. Breuer and F. Petruccioni, The Theory of Open Quantum Systems (Oxford University Press, Oxford, UK, 2002).

[4] F. Verstraete, M. M. Wolf, and J. Ignacio Cirac, Quantum computation and quantum-state engineering driven by dissipation, Nat. Phys. 5, 633 (2009).

[5] H. Carmichael, Statistical Methods in Quantum Optics 2: Non-Classical Fields, Theoretical and Mathematical Physics (Springer, Berlin, 2007).
[6] F. Minganti, A. Biella, N. Bartolo, and C. Ciuti, Spectral theory of Liouvillians for dissipative phase transitions, Phys. Rev. A 98, 042118 (2018).

[7] D. Nigro, D. Rossini, and E. Vicari, Competing coherent and dissipative dynamics close to quantum criticality, Phys. Rev. A 100, 052108 (2019).

[8] M. Kulczykowski and M. Matuszewski, Phase ordering kinetics of a nonequilibrium exciton-polariton condensate, Phys. Rev. B 95, 075306 (2017).

[9] P. Comaron, G. Dagvadorj, A. Zamora, I. Carusotto, N. P. Proukakis, and M. H. Szymańska, Dynamical Critical Exponents in Driven-Dissipative Quantum Systems, Phys. Rev. Lett. 121, 095302 (2018). 
[10] L. M. Sieberer, S. D. Huber, E. Altman, and S. Diehl, Dynamical Critical Phenomena in Driven-Dissipative Systems, Phys. Rev. Lett. 110, 195301 (2013).

[11] M. Foss-Feig, P. Niroula, J. T. Young, M. Hafezi, A. V. Gorshkov, R. M. Wilson, and M. F. Maghrebi, Emergent equilibrium in many-body optical bistability, Phys. Rev. A 95, 043826 (2017).

[12] J. Klinder, H. Keßler, M. Wolke, L. Mathey, and A. Hemmerich, Dynamical phase transition in the open Dicke model, Proc. Natl. Acad. Sci. 112, 3290 (2015).

[13] J. Lang and F. Piazza, Critical relaxation with overdamped quasiparticles in open quantum systems, Phys. Rev. A 94, 033628 (2016).

[14] D. A. Paz and M. F. Maghrebi, Critical dynamics of weaklydissipative driven systems, arXiv:1906.08278.

[15] J. Lebreuilly, A. Chiocchetta, and I. Carusotto, Pseudothermalization in driven-dissipative non-Markovian open quantum systems, Phys. Rev. A 97, 033603 (2018).

[16] M. Marcuzzi, E. Levi, W. Li, J. P. Garrahan, B. Olmos, and I. Lesanovsky, Non-equilibrium universality in the dynamics of dissipative cold atomic gases, New J. Phys. 17, 072003 (2015).

[17] J. Marino and S. Diehl, Quantum dynamical field theory for nonequilibrium phase transitions in driven open systems, Phys. Rev. B 94, 085150 (2016).

[18] P. Strack, Dynamic criticality far from equilibrium: Oneloop flow of Burgers-Kardar-Parisi-Zhang systems with broken Galilean invariance, Phys. Rev. E 91, 032131 (2015).

[19] J. T. Young, A. V. Gorshkov, M. Foss-Feig, and M. F. Maghrebi, Non-Equilibrium Fixed Points of Coupled Ising Models, Phys. Rev. X 10, 011039 (2020).

[20] J. Gelhausen and M. Buchhold, Dissipative Dicke model with collective atomic decay: Bistability, noise-driven activation, and the nonthermal first-order superradiance transition, Phys. Rev. A 97, 023807 (2018).

[21] F. Minganti, N. Bartolo, J. Lolli, W. Casteels, and C. Ciuti, Exact results for Schrödinger cats in driven-dissipative systems and their feedback control, Sci. Rep. 6, 26987 (2016).

[22] H. Goto, Universal quantum computation with a nonlinear oscillator network, Phys. Rev. A 93, 050301(R) (2016).

[23] H. Goto, Bifurcation-based adiabatic quantum computation with a nonlinear oscillator network, Sci. Rep. 6, 21686 (2016).

[24] S. E. Nigg, N. Lörch, and R. P. Tiwari, Robust quantum optimizer with full connectivity, Sci. Adv. 3, e1602273 (2017).

[25] S. Puri, C. K. Andersen, A. L. Grimsmo, and A. Blais, Quantum annealing with all-to-all connected nonlinear oscillators, Nat. Commun. 8, 15785 (2017).

[26] Z. Leghtas, S. Touzard, I. M. Pop, A. Kou, B. Vlastakis, A. Petrenko, K. M. Sliwa, A. Narla, S. Shankar, M. J. Hatridge, M. Reagor, L. Frunzio, R. J. Schoelkopf, M. Mirrahimi, and M. H. Devoret, Confining the state of light to a quantum manifold by engineered two-photon loss, Science 347, 853 (2015).

[27] C. Wang, Y. Y. Gao, P. Reinhold, R. W. Heeres, N. Ofek, K. Chou, C. Axline, M. Reagor, J. Blumoff, K. M. Sliwa, L. Frunzio, S. M. Girvin, L. Jiang, M. Mirrahimi, M. H. Devoret, and R. J. Schoelkopf, A Schrödinger cat living in two boxes, Science 352, 1087 (2016).

[28] V. Savona, Spontaneous symmetry breaking in a quadratically driven nonlinear photonic lattice, Phys. Rev. A 96, 033826 (2017).
[29] R. Rota, F. Minganti, C. Ciuti, and V. Savona, Quantum Critical Regime in A Quadratically Driven Nonlinear Photonic Lattice, Phys. Rev. Lett. 122, 110405 (2019).

[30] W. Verstraelen, R. Rota, V. Savona, and M. Wouters, Gaussian trajectory approach to dissipative phase transitions: The case of quadratically driven photonic lattices, arXiv:1912.02066.

[31] M. Foss-Feig, K. R. A. Hazzard, J. J. Bollinger, and A. M. Rey, Nonequilibrium dynamics of arbitrary-range Ising models with decoherence: An exact analytic solution, Phys. Rev. A 87, 042101 (2013); M. Foss-Feig, K. R. A. Hazzard, J. J. Bollinger, A. M. Rey, and C. W. Clark, Dynamical quantum correlations of Ising models on an arbitrary lattice and their resilience to decoherence, New J. Phys. 15, 113008 (2013).

[32] D. Jaschke, L. D. Carr, and I. de Vega, Thermalization in the quantum Ising model-Approximations, limits, and beyond, Quantum Sci. Technol. 4, 034002 (2019).

[33] W. Casteels, F. Storme, A. Le Boité, and C. Ciuti, Power laws in the dynamic hysteresis of quantum nonlinear photonic resonators, Phys. Rev. A 93, 033824 (2016).

[34] F. Vicentini, F. Minganti, R. Rota, G. Orso, and C. Ciuti, Critical slowing down in driven-dissipative Bose-Hubbard lattices, Phys. Rev. A 97, 013853 (2018).

[35] T. Fink, A. Schade, S. Höfling, C. Schneider, and A. Imamoglu, Signatures of a dissipative phase transition in photon correlation measurements, Nat. Phys. 14, 365 (2018).

[36] M. V. Medvedyeva and S. Kehrein, Power-law approach to steady state in open lattices of noninteracting electrons, Phys. Rev. B 90, 205410 (2014).

[37] L. Henriet, J. S. Douglas, D. E. Chang, and A. Albrecht, Critical open-system dynamics in a one-dimensional optical-lattice clock, Phys. Rev. A 99, 023802 (2019).

[38] R. Rota, F. Minganti, A. Biella, and C. Ciuti, Dynamical properties of dissipative XYZ Heisenberg lattices, New J. Phys. 20, 045003 (2018).

[39] Z. Cai and T. Barthel, Algebraic Versus Exponential Decoherence in Dissipative Many-Particle Systems, Phys. Rev. Lett. 111, 150403 (2013).

[40] T. Kibble, Phase-transition dynamics in the laboratory and the universe, Phys. Today 60, 47 (2007).

[41] C.-W. Liu, A. Polkovnikov, and A. W. Sandvik, Dynamic scaling at classical phase transitions approached through nonequilibrium quenching, Phys. Rev. B 89, 054307 (2014).

[42] E. Ising, Beitrag zur theorie des ferromagnetismus, Z. Phys. 31, 253 (1925).

[43] L. Onsager, Crystal statistics, I. A two-dimensional model with an order-disorder transition, Phys. Rev. 65, 117 (1944).

[44] M. J. Dunlavy and D. Venus, Critical slowing down in the two-dimensional Ising model measured using ferromagnetic ultrathin films, Phys. Rev. B 71, 144406 (2005).

[45] P. C. Hohenberg and B. I. Halperin, Theory of dynamic critical phenomena, Rev. Mod. Phys. 49, 435 (1977).

[46] W. Zhong, G. T. Barkema, D. Panja, and R. C. Ball, Critical dynamical exponent of the two-dimensional scalar $\phi^{4}$ model with local moves, Phys. Rev. E 98, 062128 (2018).

[47] At least regarding the steady-state properties.

[48] R. Rota and V. Savona, Simulating frustrated antiferromagnets with quadratically driven QED cavities, Phys. Rev. A 100, 013838 (2019). 
[49] W. Verstraelen and M. Wouters, Gaussian quantum trajectories for the variational simulation of open quantum-optical systems, Appl. Sci. 8, 1427 (2018).

[50] W. Verstraelen and M. Wouters, Temporal coherence of a photon condensate: A quantum trajectory description, Phys. Rev. A 100, 013804 (2019).

[51] K. Binder, Finite size scaling analysis of Ising model block distribution functions, Z. Phys. B 43, 119 (1981).

[52] G. N. Milstein and M. V. Tretyakov, Stochastic Numerics for Mathematical Physics (Springer-Verlag, New York, 2010).

[53] T. Kibble, Some implications of a cosmological phase transition, Phys. Rep. 67, 183 (1980).

[54] W. H. Zurek, Cosmological experiments in superfluid helium?, Nature (London) 317, 505 (1985).

[55] A. Chandran, A. Erez, S. S. Gubser, and S. L. Sondhi, KibbleZurek problem: Universality and the scaling limit, Phys. Rev. B 86, 064304 (2012).

[56] J. Sabbatini, W. H. Zurek, and M. J. Davis, Causality and defect formation in the dynamics of an engineered quantum phase transition in a coupled binary Bose-Einstein condensate, New J. Phys. 14, 095030 (2012).

[57] J. Dziarmaga, Dynamics of a quantum phase transition and relaxation to a steady state, Adv. Phys. 59, 1063 (2010).

[58] A. Polkovnikov, K. Sengupta, A. Silva, and M. Vengalattore, Colloquium: Nonequilibrium dynamics of closed interacting quantum systems, Rev. Mod. Phys. 83, 863 (2011).

[59] D. Jaschke, K. Maeda, J. D. Whalen, M. L. Wall, and L. D. Carr, Critical phenomena and Kibble-Zurek scaling in the long-range quantum Ising chain, New J. Phys. 19, 033032 (2017).

[60] P. Silvi, G. Morigi, T. Calarco, and S. Montangero, Crossover from Classical to Quantum Kibble-Zurek Scaling, Phys. Rev. Lett. 116, 225701 (2016).

[61] J. Cardy, Finite-Size Scaling (Elsevier, New York, 1988).
[62] More precisely, $v_{a}$ relates to the energy scales of individual Hamiltonian processes and dissipation rate, which we all take of order 1 .

[63] The same classical model can have different simulation algorithms (update rules) with other values of $z$ [41].

[64] R. Puebla, O. Marty, and M. B. Plenio, Quantum Kibble-Zurek physics in long-range transverse-field Ising models, Phys. Rev. A 100, 032115 (2019).

[65] A. Dutta, A. Rahmani, and A. del Campo, Anti-Kibble-Zurek Behavior in Crossing the Quantum Critical Point of a Thermally Isolated System Driven by a Noisy Control Field, Phys. Rev. Lett. 117, 080402 (2016).

[66] R. Puebla, A. Smirne, S. F. Huelga, and M. B. Plenio, Universal anti-Kibble-Zurek scaling in fully-connected systems, arXiv:1911.06023.

[67] F. Barahona, On the computational complexity of Ising spin glass models, J. Phys. A: Math. Gen. 15, 3241 (1982).

[68] A. Marandi, Z. Wang, K. Takata, R. L. Byer, and Y. Yamamoto, Network of time-multiplexed optical parametric oscillators as a coherent Ising machine, Nat. Photonics 8, 937 (2014).

[69] T. Inagaki, Y. Haribara, K. Igarashi, T. Sonobe, S. Tamate, T. Honjo, A. Marandi, P. L. McMahon, T. Umeki, K. Enbutsu, O. Tadanaga, H. Takenouchi, K. Aihara, K.-i. Kawarabayashi, K. Inoue, S. Utsunomiya, and H. Takesue, A coherent Ising machine for 2000-node optimization problems, Science 354, 603 (2016).

[70] P. L. McMahon, A. Marandi, Y. Haribara, R. Hamerly, C. Langrock, S. Tamate, T. Inagaki, H. Takesue, S. Utsunomiya, K. Aihara, R. L. Byer, M. M. Fejer, H. Mabuchi, and Y. Yamamoto, A fully programmable 100-spin coherent Ising machine with all-to-all connections, Science 354, 614 (2016).

[71] O. Kyriienko, H. Sigurdsson, and T. C. H. Liew, Probabilistic solving of $n p$-hard problems with bistable nonlinear optical networks, Phys. Rev. B 99, 195301 (2019). 